\title{
ARTICLE
}

\section{Liquid-state carbon-13 hyperpolarization generated in an MRI system for fast imaging}

\author{
A.B. Schmidt ${ }^{1}$, S. Berner ${ }^{1,2,3}$, W. Schimpf ${ }^{1}$, C. Müller ${ }^{1,2,3}$, T. Lickert ${ }^{4}$, N. Schwaderlapp ${ }^{1}$, S. Knecht ${ }^{1}$, J.G. Skinner ${ }^{1}$,
} A. Dost ${ }^{1}$, P. Rovedo', J. Hennig ${ }^{1}$, D. von Elverfeldt ${ }^{1} \&$ J.-B. Hövener ${ }^{1,2}$

Hyperpolarized (HP) tracers dramatically increase the sensitivity of magnetic resonance imaging (MRI) to monitor metabolism non-invasively and in vivo. Their production, however, requires an extra polarizing device (polarizer) whose complexity, operation and cost can exceed that of an MRI system itself. Furthermore, the lifetime of HP tracers is short and some of the enhancement is lost during transfer to the application site. Here, we present the production of HP tracers in water without an external polarizer: by Synthesis Amid the Magnet Bore, A Dramatically Enhanced Nuclear Alignment (SAMBADENA) is achieved within seconds, corresponding to a hyperpolarization of $\sim 20 \%$. As transfer of the tracer is no longer required, SAMBADENA may permit a higher polarization at the time of detection at a fraction of the cost and complexity of external polarizers. This development is particularly promising in light of the recently extended portfolio of biomedically relevant para-hydrogentracers and may lead to new diagnostic applications.

\footnotetext{
${ }^{1}$ Department of Radiology, Medical Physics, Medical Center-University of Freiburg, Faculty of Medicine, University of Freiburg, Breisacherstrasse 60a, Freiburg 79106, Germany. ${ }^{2}$ German Consortium for Cancer Research (DKTK), Im Neuenheimer Feld 280, Heidelberg 69120, Germany. ${ }^{3}$ German Cancer Research Center (DKFZ), Im Neuenheimer Feld 280, Heidelberg 69120, Germany. ${ }^{4}$ Division Hydrogen Technologies, Fraunhofer Institute for Solar Energy Systems (ISE), Heidenhofstraße 2, Freiburg 79110, Germany. Correspondence and requests for materials should be addressed to J.-B.H. (email: jan.hoevener@uniklinik-freiburg.de).
} 
W ithin the past decades, magnetic resonance imaging (MRI) has become an indispensable tool for medical diagnostics. Because of its low sensitivity, however, the only nucleus used in clinical routine is hydrogen $\left({ }^{1} \mathrm{H}\right)$, which is the most abundant element in vivo $(\sim 80 \mathrm{M})$ and holds the greatest gyromagnetic ratio of all stable nuclei $(42 \times$ $\left.2 \pi \mathrm{MHz} \mathrm{T}^{-1}\right)$. But even for ${ }^{1} \mathrm{H}$, the polarization, which is the fraction of all spins contributing to the MR signal, does not exceed 3 p.p.m. in a magnetic field of $1 \mathrm{~T}$. All other stable nuclei have a lower polarization and much lower abundance in vivo.

Still, the need for a non-invasive tool for chemical analysis in vivo, for example to measure the metabolism and treatment response of tumors ${ }^{1}$, is great. It appears that this need is precisely met by magnetic resonance (MR) spectroscopy, which is very successful for chemical analysis in vitro. However, low sensitivity makes its use in clinical routines very limited ${ }^{2}$. Nuclear hyperpolarization (HP) promises to overcome this hurdle and has enabled MR signal enhancement by several orders of magnitude ${ }^{3}$. The MR signal of most X-nuclei (nuclei other than protons) in vivo is low and thus both distribution and metabolism of magnetically labelled or hyperpolarized X-nuclei can be observed without significant background, similar to positron emission tomography. The first human studies of this method have shown very promising results ${ }^{4,5}$ and more are underway.

The most prominent techniques for the production of hyperpolarized ${ }^{13} \mathrm{C}$-tracers are dissolution dynamic nuclear polarization $(\mathrm{dDNP})^{6}$ and parahydrogen $\left(\mathrm{H}_{2}\right)$ and synthesis allows dramatically enhanced nuclear alignment (PASADENA) ${ }^{7}$, also known as $\mathrm{pH}_{2}$-induced polarization (PHIP) ${ }^{8}$. dDNP achieves liquid-state polarization by transferring polarization from electrons to nuclei in the solid state at a temperature of a few $\mathrm{K}$ and in a strong magnetic field of a few $\mathrm{T}$, followed by rapid dissolution. PHIP, in contrast, is the only technique to date that allows polarization to take place in the liquid state, either by hydrogenation ${ }^{7}$ or reversible exchange with $\mathrm{pH}_{2}$ (ref. 9). While dDNP is the only technique commercially available and used for human applications, it is associated with rather high cost, a relatively low output and long polarization cycles, although many promising attempts are being pursued to overcome these shortcomings ${ }^{10-12}$. PHIP, in contrast, is more cost efficient, very fast and requires less hardware. A hitherto substantial drawback of this technique-its limited portfolio of tracers - may have been overcome by the discovery of new agents ${ }^{13-15}$ and side-arm hydrogenation PHIP (PHIP-SAH), which has enabled the polarization of acetate and pyruvate ${ }^{16}$. Both methods share the disadvantage that the HP-tracer has to be produced in a dedicated polarizing device (polarizer) outside of the MRI system $3,6,17-29$ During the ensuing transfer through varying magnetic fields to the application site, inevitably, some of the polarization is lost to the $T_{1}$ decay towards thermal equilibrium.

Here, we present a new method that achieves ${ }^{13} \mathrm{C}-\mathrm{HP}$ in excess of $20 \%$ in aqueous solution within seconds in the bore of a commercial MRI system. No external polarizer is needed-the only items that are required, in addition to an MRI scanner, are $p \mathrm{H}_{2}$, a reaction chamber, some valves and a digital output to control the process. By Synthesis Amid the Magnet Bore, A Dramatically Enhanced Nuclear Alignment (SAMBADENA) is obtained. By producing a sterile HP-tracer in situ, within the bore of an MRI, at the application site and at high field, this new method addresses some of the aforementioned shortcomings of dDNP and PHIP.

\section{Results}

SAMBADENA produces high and reproducible in-bore ${ }^{13} \mathrm{C}-\mathrm{HP}$. A maximum theoretical polarization yield of $48.7 \%$ was simulated for $1-{ }^{13} \mathrm{C}$ hydroxyenthyl-propionate (HEP), a tracer that was previously used for in vivo angiography ${ }^{27,30}$. The corresponding evolution intervals for the spin-order-transfer (SOT) sequence l-PH-INEPT + (longitudinal-para-hydrogen-insensitive nuclei enhancement by polarization transfer) were $t_{1}=69.8 \mathrm{~ms}$ and $t_{2}=38.9 \mathrm{~ms}$ (total $108.7 \mathrm{~ms}$ ). Several other polarization maxima were found but required longer evolution intervals (Fig. 1). The polarization yield was found to be relatively stable with respect to errors of J-couplings, pulses and evolution times (Fig. 1).

Experimentally, HP was produced within an MRI system by using the available hardware of the system itself (Fig. 2). The signal that was directly produced by the $\mathrm{PH}-\mathrm{INEPT}+$ sequence was quantified and corresponded to a nuclear polarization of $P=24 \%$, approximately half of the theoretical maximum. This polarization level corresponds to an enhancement factor of $\eta \approx 40,000$ for $13 \mathrm{C}$ at $7 \mathrm{~T}$ (hydrogenation time $t_{\mathrm{h}}=4 \mathrm{~s}, 5.54 \mathrm{mM}$ $\mathrm{HEP}$ in $\mathrm{H}_{2} \mathrm{O}$; Fig. 3).

The inter-day yield and reproducibility of SAMBADENA was quantified in nine experiments to $P \approx(21 \pm 2) \%$ with $5.54 \mathrm{mM}$ $\mathrm{HEP}$ in $\mathrm{H}_{2} \mathrm{O}$ (Fig. 4).

Production of longitudinal hyperpolarization by l-PH-INEPT + . While a polarization of the order of few per cent is sufficient for biomedical use $\mathrm{u}^{3,31}$, the short-lived transversal polarization generated by the PH-INEPT + sequence is not suited for

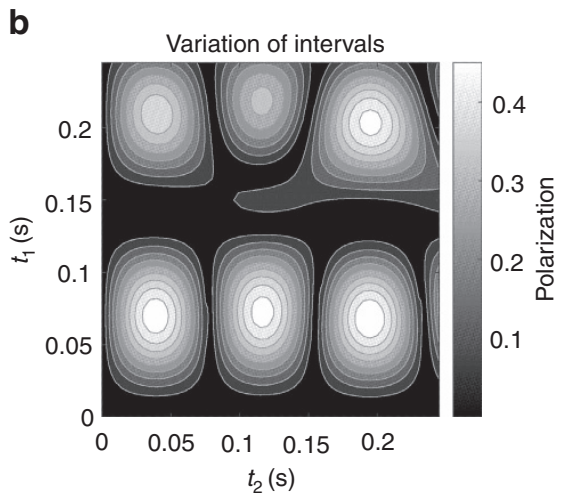

Figure 1 | Simulated stability and intervals of I-PHINEPT for SAMBADENA. The resulting polarization of the SOT sequence I-PH-INEPT + (longitudinalparahydrogen-insensitive nuclei enhancement by polarization transfer) was simulated as function of erroneous J-couplings and flip angles (a) and as function of the evolution intervals (b). J represents the J-couplings of the simplified 3-spin- $1 / 2$ system of the tracer and $\alpha$ represents the flip angle of the pulses of the sequence; both values were varied by $\pm 15 \%$. The values of $t_{1}$ and $t_{2}$ denote the free evolution intervals between the effective pulses of the SOT sequence (see Fig. 8). 


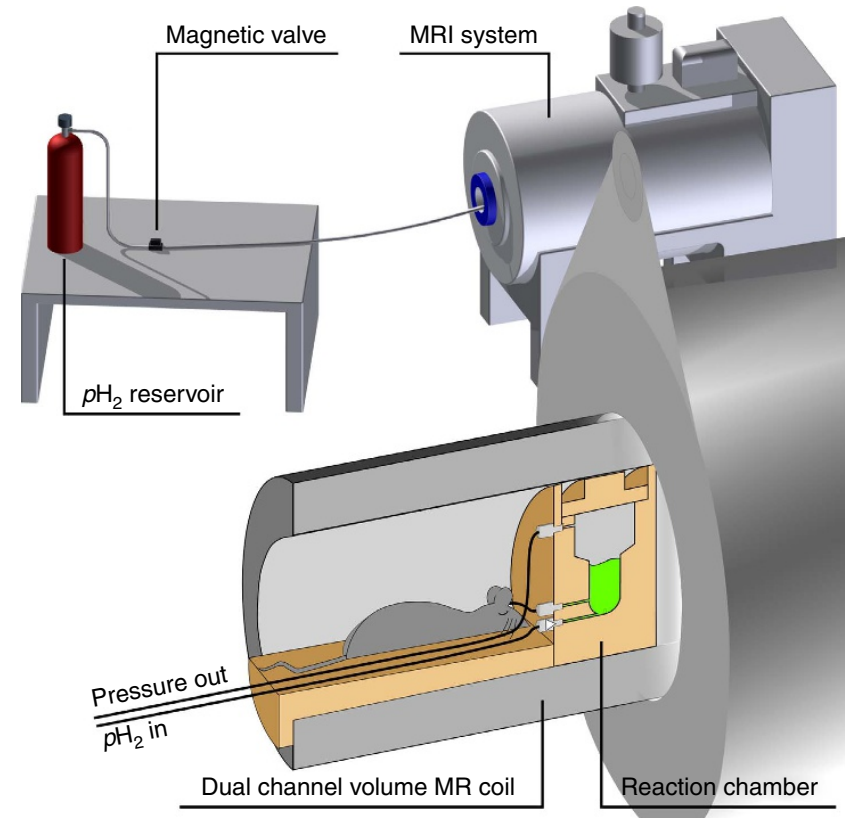

Figure 2 | HP without a polarizer: Schematic view of the experimental setup, where the HP-tracer is produced in situ within the MRI close to the application site. The essential components comprise the reaction chamber, valves, tubing, $\mathrm{pH}_{2}$ and the MRI system. The latter is used to transfer the spin order and to detect the hyperpolarized signal.

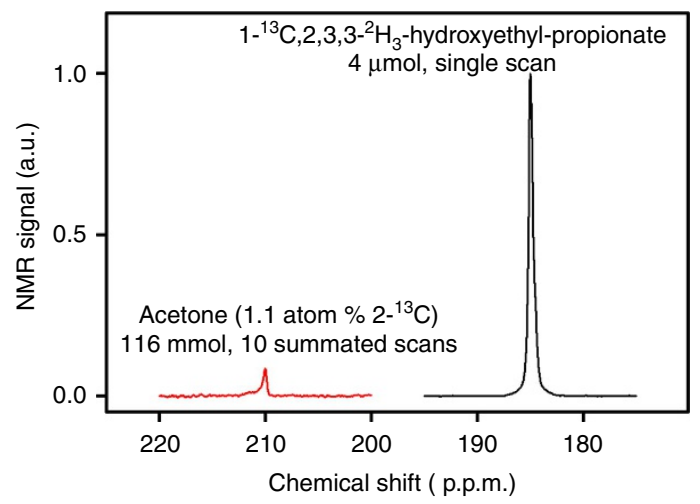

Figure 3 | ${ }^{13} \mathbf{C}$-HP generated inside an MRI system. Non-localized ${ }^{13} \mathrm{C}$-spectra of thermally polarized acetone at natural abundance (red) and ${ }^{13} \mathrm{C}$-HEP in aqueous solution (black) hyperpolarized by SAMBADENA within an MRI system. The polarization yield was quantified to $\sim 24 \%$, which corresponds to a $\sim 40,000$-fold enhancement of the thermal ${ }^{13} \mathrm{C}$-polarization which amounts to $\sim 6$ p.p.m. at $7 \mathrm{~T}$.

in vivo application-longitudinal magnetization is required instead. As predicted by Bär et al. ${ }^{32}$, the l-PH-INEPT + sequence enabled the production of longitudinal magnetization hyperpolarized to a similar level, $P=(19 \pm 2) \%$ (detected $2 \mathrm{~s}$ after generation, for $5.54 \mathrm{mM}$ HEP in $\mathrm{D}_{2} \mathrm{O}$ ) (Fig. 5). This magnetization decayed much slower with $T_{1}$ (instead of $T_{2}$ ) and enabled other experiments as described in the following.

SAMBADENA enables magnetization storage at high field. The $T_{1}$ of HEP dissolved in $\mathrm{D}_{2} \mathrm{O}$ was measured to $(79 \pm 2) \mathrm{s}$ at $7 \mathrm{~T}$ (Fig. 5), which is more than the $70 \mathrm{~s}$ reported at $4.7 \mathrm{~T}$ (ref. 26). In degassed and deionized $\mathrm{H}_{2} \mathrm{O}$, the $T_{1}$ of HEP at $7 \mathrm{~T}$ was measured to $(75 \pm 5) \mathrm{s}$, which is less than $(101 \pm 7) \mathrm{s}$ reported at $48 \mathrm{mT}$ (ref. 19). These long relaxation times are advantageous with

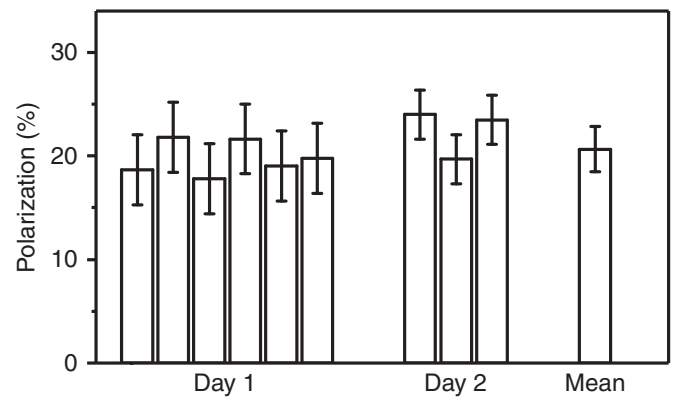

Figure 4 | SAMBADENA reproducibility: A mean polarization of $P=(21 \pm 2) \%$ was achieved in nine experiments on 2 days. Error bars correspond to the s.d. of the respective day or, in case of the mean, all experiments (reaction temperature $T \approx 80^{\circ} \mathrm{C}$, hydrogenation time $t_{\mathrm{h}}=4 \mathrm{~s}$, concentration of substrate $\mathrm{C}_{\text {HEP }}=5.54 \mathrm{mM}$ ).

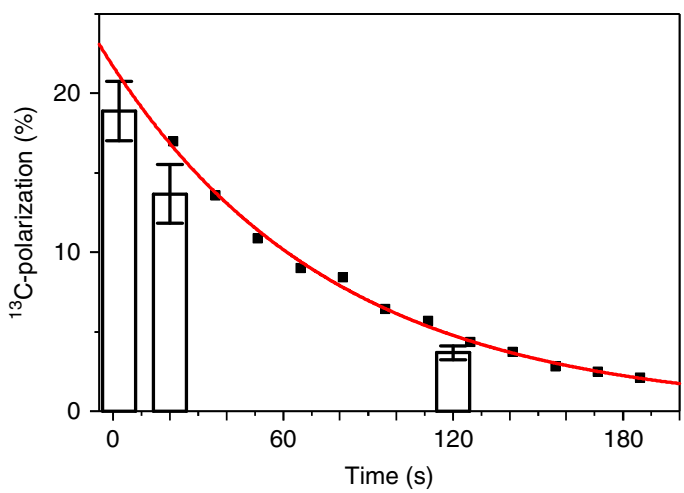

Figure 5 | The lifetime of the polarization is long at high field. The decay of one sample of hyperpolarized ${ }^{13} \mathrm{C}$-HEP was monitored by acquiring 12 non-localized spectra with a repetition time of $T_{R}=15 \mathrm{~s}$ and a small flip angle of $9^{\circ}$ (squares). A mono-exponential decay function (line) was fitted to the data and yielded $T_{1} \sim(79 \pm 2) \mathrm{s}$. A correction factor-described in the methods-was applied to each data point to compensate for the fact that each measurement pulse consumes a finite amount of the magnetization. The $T_{1}$ determined from the low flip angle measurement correlates well to the polarizations that were detected by full, $90^{\circ}$ excitations 0,20 and $120 \mathrm{~s}$ after the HP. For each time point, the mean of three measurements (bars) is shown, and error bars indicate the s.d. The tracer concentration was $5.54 \mathrm{mM}$ in $\mathrm{D}_{2} \mathrm{O}$.

respect of the further use of the tracer. If, for example, $20 \mathrm{~s}$ were to be used for the injection of the tracer in vivo, only about one fifth of the initial polarization would be lost.

Optimized reaction conditions. As expected and reported before $^{18}$, the hydrogenation time had a strong impact on the HP yield: the highest polarizations were observed between $t_{\mathrm{h}}=4-8 \mathrm{~s}$ (Fig. 6). Note that considerable polarization, about half of the maximum, was observed after hydrogenation periods $t_{\mathrm{h}}$ as long as $15 \mathrm{~s}$. The slow decay is likely attributed to the decay of the paraorder of the hydrogens that were added to the tracer. Note that no refocusing or decoupling pulses were applied during $t_{\mathrm{h}}$.

The apparent build-up hydrogenation time constant of the catalysis $\left(T_{\text {cat }}\right)$ and para-order relaxation constant $\left(T_{\text {para }}\right)$ were estimated to $T_{\text {cat }}=(1.6 \pm 0.9) \mathrm{s}$ and $T_{\text {para }}=(16 \pm 6) \mathrm{s}$, respectively, by fitting a model to the experimental data (equation (2) in Methods). The onset of the hydrogenation reaction $\left(t_{0}\right)$ extracted from the fit was $t_{0}=(1.0 \pm 0.5) \mathrm{s}$. This value reflects the finite time required for the hydrogen being delivered to the reactor, to enter the solution, to build-up pressure and to start the reaction. 
It is supported by a high-speed video of the hydrogenation reaction, where the bubbling came to a stop $\sim 1 \mathrm{~s}$ after the injection, indicating an equilibration of the pressure (Supplementary Movie 1). Disregarding the contribution of relaxation, the asymptotic maximum polarization level extracted from the fit was $(27 \pm 5) \%$ (goodness of the fit: $\mathrm{R}^{2}=0.7$, concentrations of HEP and catalyst of $c_{\mathrm{HEP}}=5.5 \mathrm{mM}$ and $c_{\text {cat }}=2.1 \mathrm{mM}$ ). The deviation to the theoretical maximum may be attributed to noise in the data, relaxation before and during the SOT-sequence itself or experimental imperfections.

This data indicates that after $t_{\mathrm{h}}=4 \mathrm{~s},(85 \pm 18) \%$ of all molecules are hydrogenated. Since $100 \%$ hydrogenation is assumed for the quantification of the polarization yield, it is likely that the reported polarization is underestimated. Note that $T_{\text {cat }}, T_{\text {para }}$, maximum polarization level and the optimal $t_{\mathrm{h}}$ are expected to be dependent on concentrations of the precursor and catalyst.

Hyperpolarization at elevated tracer concentrations. For any biomedical application, highly concentrated, highly hyperpolarized samples are needed to achieve sufficient signal. By variation of $t_{\mathrm{h}}$, the catalyst concentration $\left(c_{\mathrm{cat}}\right)$ and reaction pressure $(p)$, the polarization achieved for $80 \mathrm{mM}$ tracer was increased from 3.4 to $7.1 \%$ and eventually $\sim 13 \%$ (Table 1 and Supplementary Fig. 1). It must be noted, though, that the last measurement could not be repeated because the pressure exceeded the specifications of the current instrumentation and caused damage to the equipment. In addition, $P \approx 17 \%$ was readily achieved in a sample of $22 \mathrm{mM}, 3.5 \mathrm{~cm}$ off the isocentre of the magnet.

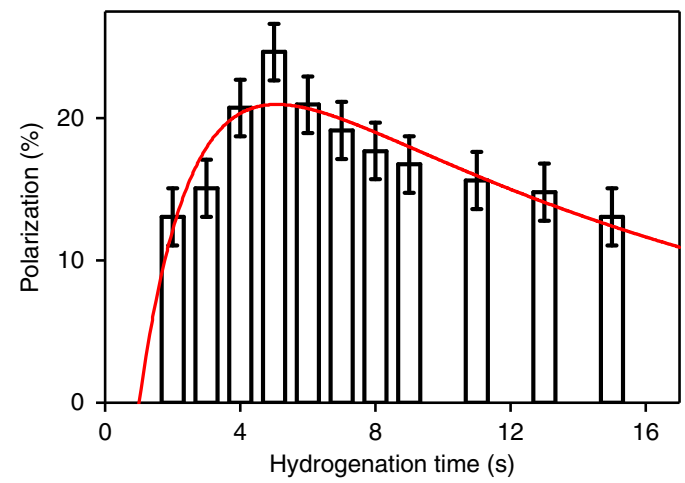

Figure 6 | Hydrogenation time affects polarization. Mean ${ }^{13} \mathrm{C}-\mathrm{HP}$ (columns) and s.d. (error bars) of HEP (1- ${ }^{13} \mathrm{C}$ HEP) as a function of hydrogenation time $\left(t_{h}\right)$ and fit (line). In agreement with earlier reports ${ }^{18}$ the optimum $t_{h}$ was between $4-8 \mathrm{~s}$. The time constants for the catalytic hydrogenation reaction, $T_{\text {cat }}=(1.6 \pm 0.9) \mathrm{s}$, and the para-order relaxation, $T_{\text {para }}=(16 \pm 6) \mathrm{s}$, were extracted from a model fitted to the data. The model is described in Methods (equation (2)). Each time point was measured three times.
SAMBADENA and $e x$ vivo ${ }^{13} \mathrm{C}-\mathrm{MRI}$. To demonstrate the feasibility of simultaneous HP and MRI, HEP was hyperpolarized by SAMBADENA within seconds, injected into a rat post mortem and ${ }^{13} \mathrm{C}-\mathrm{MRI}$ was acquired (rattus rattus, 7 days old). The rat was placed next to the reaction chamber and positioned in the sensitive volume of the coil within the magnet (Fig. 7a,b). In contrast to all other experiments, in this setting, the reaction chamber was not in the isocentre, but at a distance of $3.5 \mathrm{~cm}$ along the $z$ axis of the magnet. At this position, the SOT sequence was still efficient, as demonstrated by an HP of $17 \%$ that was achieved for $22 \mathrm{mM} \mathrm{HEP}$ in $700 \mu \mathrm{l}$ aqueous solution in the reactor (Table 1).

For ex vivo MRI, a catheter was connected to the outlet of the reaction chamber and inserted into the thorax of the rat. The HP-experiment was repeated and $\sim 330 \mu$ l of the hyperpolarized tracer were injected through the catheter into the rat without leaving the magnet. Sub-second ${ }^{13} \mathrm{C}-\mathrm{MRI}$ was acquired and strong, hyperpolarized signal was observed (Fig. 7c). The co-registration with a $T_{2}$-weighted ${ }^{1} \mathrm{H}$-MRI (Fig. $\left.7 \mathrm{~d}, \mathrm{e}\right)$ showed that the ${ }^{13} \mathrm{C}$-signal was localized around the heart of the animal and within the reaction chamber, with a maximum ${ }^{13} \mathrm{C}$-signal-tonoise ratio (SNR) of 113 and 111, respectively (Supplementary Fig. 2 and Supplementary Table 1). In the same scan, the SNR in the model solution was quantified to 9. A Zero-Echo-Time (ZTE) $\mathrm{MRI}^{33}$ was acquired afterwards to depict the entire setup (Fig. 7f).

\section{Discussion}

SAMBADENA enables the HP of ${ }^{13} \mathrm{C}$-labelled tracers to high levels, in aqueous solution, within seconds and within the magnet bore next to the application site. For the first time, high ${ }^{13} \mathrm{C}-\mathrm{HP}$ was achieved without a dedicated polarizer-the hardware of an MRI system was used instead. This method circumvents some of the shortcomings that are associated with current ${ }^{13} \mathrm{C}-\mathrm{HP}$ methods, foremost the need of a dedicated, expensive and complex external polarizer and lengthy transfer of the sample. Other challenges persist and are discussed in the following.

Obtaining a pure solution devoid of the catalyst is a persisting challenge for all $\mathrm{pH}_{2}$-based $\mathrm{HP}$ methods, although strong progress was reported recently by using either heterogeneous catalysts $^{34-37}$ or a biphasic approach ${ }^{38}$. The latter appears to be particularly promising with respect to the implementation in the presented setup, and heterogeneous catalysts are currently being investigated in our laboratory.

Whereas other HP methods require an extra polarizer device whose complexity, operation and cost can exceed that of the MRI scanner itself, the additional equipment needed here is very little. The only extra component that is needed inside the magnet is a reaction chamber. In the experiments presented here, the bulk reactor occupies less than one-third of the imaging field of view (FOV). A significant reduction in size appears feasible because the actual inner volume of the reactor is less than $1 / 100$ of the FOV

Table 1 | Experimental parameters and corresponding polarization yields.

\begin{tabular}{|c|c|c|c|c|c|}
\hline P (\%) & $p\left(p \mathrm{H}_{2}\right)$ (bar) & $c_{\text {cat }}(\mathrm{mM})$ & $c_{\text {HEP }}(\mathrm{mM})$ & $t_{h}(s)$ & Solvent \\
\hline 3.4 & 15 & 2.1 & 80 & 4 & $\mathrm{D}_{2} \mathrm{O}$ \\
\hline 7.1 & 15 & 2.1 & 80 & 8 & $\mathrm{D}_{2} \mathrm{O}$ \\
\hline 13 & 30 & 4.2 & 80 & 8 & $\mathrm{H}_{2} \mathrm{O}$ \\
\hline 17 & 15 & 4.2 & 22 & 5 & $\mathrm{H}_{2} \mathrm{O}$ \\
\hline
\end{tabular}




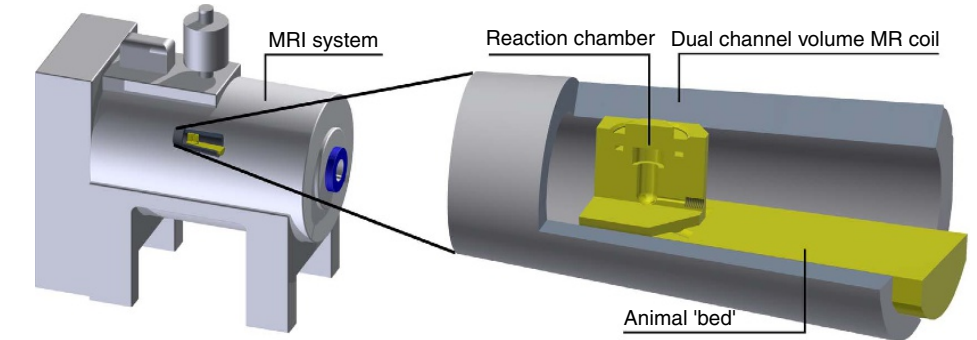

a $3 \mathrm{D}$ view

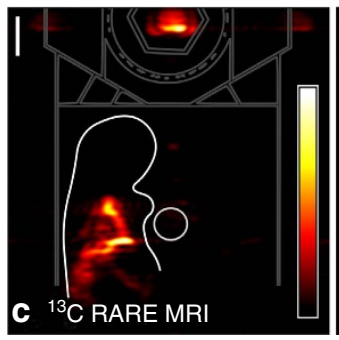

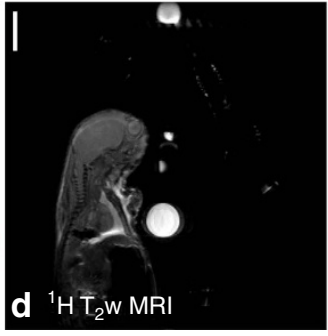

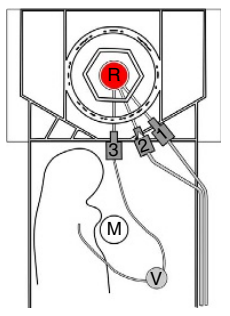

b Schematic setting

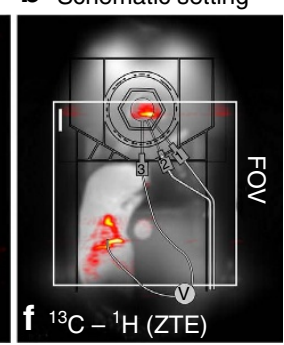

Figure 7 I Simultaneous ${ }^{\mathbf{1 3}} \mathbf{C}$-HP and ex vivo imaging: The compact design of the setup enabled SAMBADENA next to a small rat post mortem in the sensitive volume of the magnet $(\mathbf{a}, \mathbf{b})$. Within seconds, the ${ }^{13} \mathrm{C}$-tracer HEP was polarized to $\sim 17 \%$ at a concertation of 22 mM. Without leaving the magnet, $\sim 330 \mu \mathrm{l}$ of the tracer were injected into the rat, and strong ${ }^{13} \mathrm{C}$ signal with a maximum SNR of 113 was observed by MRI acquired in $0.5 \mathrm{~s}$ (c). Subsequently, a $T_{2}$-weighted ${ }^{1} \mathrm{H}$-MRI (d) and ${ }^{1} \mathrm{H}$ Zero-Echo-Time (ZTE) MRI were recorded. Co-registration of ${ }^{1} \mathrm{H}$ and ${ }^{13} \mathrm{C}$-data revealed the location of the tracer, in the reaction chamber and surrounding the heart of the animal (e,f). The isocenter of the magnet is the center of the images. $R$, reactor; FOV, field of view; $M$, Model Solution $\mathrm{M} 3 ; 1,2$, 3: lines for pressure relieve, $\mathrm{pH}_{2}$ supply and $\mathrm{pH}_{2}$ injection, respectively.

$\left(2 \mathrm{~cm}^{3}\right.$ versus $\left.342 \mathrm{~cm}^{3}\right)$. The only requirement for the MRI system is a ${ }^{13} \mathrm{C}$ channel, which is necessary for ${ }^{13} \mathrm{C}$-MRI in general.

A relatively large volume transmitter is advantageous to enable simultaneous application of the SOT and MRI sequence. A dedicated pickup coil at the region of interest (ROI) may assure a high sensitivity while the homogeneous excitation of the large volume coil (covering both chamber and animal) would be maintained. To improve the accuracy of the applied pulse sequences, two different shim settings were used; one for the HP and one for the imaging.

SAMBADENA may be simplified further by using the digital outputs of the MRI system to control the valves; its implementation on any commercial multinuclear MRI system appears feasible. Other $\mathrm{pH}_{2}$-based approaches are much less cost intensive than $\mathrm{dDNP}$, but still require an external polarizer device with the associated cost and complexity ${ }^{17-23,26-29}$.

While the actual HP process is executed with a single button, the production of $\mathrm{pH}_{2}$ and precursor solution requires some training. In the future, both $\mathrm{pH}_{2}$ and solution may be prepared elsewhere and shipped to the application site. At this stage, the catalyst-substrate solution is filled manually into the reactor outside the scanner. We envision that future implementations will feature in-bore loading, automatic heating and likely automated injection $^{18}$.

Any preparation of the hyperpolarized tracer, such as cooling or quality control, would take place at the elevated field of the host MRI system where the $T_{1}$ is long (here: $\sim 80 \mathrm{~s}$ ). Note, however, that any human application would require fast and comprehensive testing of the solution before injection, to ensure the safety of the patient, which is challenging especially in the magnet bore.

The HP levels reached $-\sim 20 \%$ for a tracer concentration of 6 and $22 \mathrm{mM}$, and $13 \%$ for $80 \mathrm{mM}$-are likely sufficient for a meaningful in vivo application, in particular because no lengthy transfer of the tracer through varying magnetic fields is necessary.

The SOT-sequence 1-PH-INEPT + was implemented for the first time, and about half of the theoretically achievable polarization yield was observed $(P=(19 \pm 2) \%)$. Other sequences with a higher theoretical yield appear feasible and may improve the polarization as well.

Fast hydrogenation at elevated temperature and high pressure was found to be essential for a high polarization (Fig. 2 and Supplementary Fig. 1). The catalytic hydrogenation time constant extracted from experimental data indicated that $(85 \pm 18) \%$ of a $5.5 \mathrm{mM}$ precursor solution were hydrogenated after a $4 \mathrm{~s}$ reaction at 15 bar and $\sim 80^{\circ} \mathrm{C}$. Especially with respect to a high substrate concentration, an even higher pressure and temperature is expected to improve the yield further (see Supplementary Fig. 1).

Polarization losses may be attributed to relaxation before, during and after the polarization transfer, imperfect pulses, timings (or erroneous J-coupling constants) and incomplete hydrogenation. We observed that the reaction chamber distorted the line shape of a model solution, which may also cause polarization loss (see Supplementary Figs 3 and 4). Note that $100 \%$ hydrogenation and $\mathrm{pH}_{2}$ enrichment was assumed for the quantification, which results in some underestimation of the reported polarization.

The technical feasibility of using the MRI simultaneously for the HP and subsequent imaging was demonstrated by an ex vivo experiment (Fig. 7). Although there appears to be no fundamental hurdle for a preclinical in vivo application, the following aspects have to be taken into account: First, the equipment to support the animal (anaesthesia, vital sign monitoring, heating) have to fit into the bore along with the animal and the reactor. This issue may be addressed by employing a smaller reactor designed to be used with a commercial animal bed. Second, the injection needs to be well controlled regarding flow, pressure and total volume. An automated injection system for dDNP that was already described and evaluated positively in many in vivo experiments ${ }^{39}$ may be used here. Third, unwanted heating of the animal (by the reactor) should be avoided, and the HP-solution needs to be cooled to temperature that is acceptable for injection. This first implementation was not designed with an in vivo demonstration in mind, but the temperature at the position of the animal is close to the required range (measured as 42 to $37^{\circ} \mathrm{C}$ as a function of distance from the outside wall of the reactor, see Supplementary 
Fig. 5). The temperature at the position of the animal may be reduced further by adding insulation and/or using a different method of heating. As for the temperature of the solution, an elevated temperature is required only at the time of the hydrogenation (to provide fast catalysis, a high polarization yield and sterilize the tracer solution, see Supplementary Note 1). The temperature of the tracer exiting the catheter used in vitro was measured to $(43 \pm 3)^{\circ} \mathrm{C}$ and was further reduced to $(32 \pm 3)^{\circ} \mathrm{C}$ by guiding the catheter through iced water (Supplementary Table 2). Thus, it appears feasible to reach an acceptable temperature within seconds. Current work focuses on adapting SAMBADENA for the HP of biomolecules to conduct metabolic studies in vivo.

A major limitation of $\mathrm{pH}_{2}$-based methods was the hitherto restricted portfolio of relevant tracers. However, this hurdle may have been overcome by the HP of pyruvate and acetate ${ }^{16}$, phospholactate ${ }^{13-15}$, and (diethyl-) succinate ${ }^{1,40-43}$ with $\mathrm{pH}_{2}$. Current work focuses on adapting SAMBADENA to these tracers.

Compared with other methods, the HP of a liquid-state tracer next to the application site may enable the delivery of higher polarization in vivo at the time of detection, especially for agents with short relaxation time $T_{1}$ (such as glucose), and enable better resolution of metabolites with hitherto low SNR.

Given its simplicity, its high polarization yield, fast production and low cost, SAMBADENA may have an important impact on the research of HP tracers, which hold the potential to revolutionize the use of MRI in modern diagnostics.

\section{Methods \\ Parahydrogen production. $\mathrm{pH}_{2}$ was enriched to $\sim 95 \%$ similar as descried} previously and stored in aluminium bottle to be used on demand ${ }^{44}$.

Experimental setup. Measurements were performed using a $7 \mathrm{~T}$ preclinical small animal MRI system (Biospec 7/20, PV5.1, Bruker, Germany), using a dual-resonant ${ }^{1} \mathrm{H}-{ }^{13} \mathrm{C}$ transmit-receive volume coil (Rapid, Germany; Fig. 2).

A reaction chamber with an inner volume of $\sim 2 \mathrm{ml}$ was custom-made from polysulfone (PSU 1000) to allow a high hydrogenation temperature $\left(\sim 80^{\circ} \mathrm{C}\right)$ and pressure $(\sim 15$ bar $)$. Tubes were connected to the inlet at the bottom $\left(1 / 8^{\prime \prime} \times 1 / 16^{\prime \prime}\right.$, PTFE, SCP GmbH, Germany) and outlet at the top $(1 / 16 " \times 0.75 \mathrm{~mm}$, PTFE, SCP $\mathrm{GmbH}$, Germany) to inject and release $\mathrm{pH}_{2}$, respectively. Both tubes were connected to magnetic valves (type 0124, Bürkert, Germany) outside of the MR system. The valves were controlled by a custom-written software (MATLAB, MathWorks, USA) using the digital outputs of a data acquisition board (DAQ 6125 , National Instruments, USA). A second reactor was used for hydrogenation experiments (Fig. 6) and ex vivo MRI experiments, where an additional outlet at the bottom served to inject the tracer into the animal (Fig. 7).

Preparation of the samples. A rhodium-based catalyst was prepared fresh by mixing a biphosphine ligand (1,4-bis-[(phenyl-3-propane sulfonate) phosphine] butane disodium salt, Q36333, MW $=562.53 \mathrm{~g} \mathrm{~mol}^{-1}$, Sigma Aldrich, MO, USA) with a rhodium complex (bis(norbornadiene)rhodium (I) tetrafluoroborate, $\mathrm{MW}=373.99 \mathrm{~g} \mathrm{~mol}^{-1}$, CAS 36620-11-8, StremChemicals, MA, USA) in degassed $\mathrm{D}_{2} \mathrm{O}$ (Deuterium oxide, 99.9 atom \% D, CAS 7789-20-0, Sigma Aldrich, MO, USA) or degassed and deionized $\mathrm{H}_{2} \mathrm{O}$ (ref. 17).

In contrast to previous reports ${ }^{17,26}$, the rhodium moiety was dissolved in warm $\mathrm{H}_{2} \mathrm{O} / \mathrm{D}_{2} \mathrm{O}\left(\sim 60^{\circ} \mathrm{C}\right)$ instead of acetone.

$1-{ }^{13} \mathrm{C}, 2,3,3-{ }^{2} \mathrm{H}_{3}$-hydroxyethyl-acrylate (HEA, $99 \%{ }^{13} \mathrm{C}, \mathrm{MW}=120.13 \mathrm{~g} \mathrm{~mol}^{-1}$, CAS: 1216933-17-3, Sigma Aldrich, USA) was added to the solution. During the HP experiment, $p \mathrm{H}_{2}$ was added to HEA and $1-{ }^{13} \mathrm{C}, 2,3,3-{ }^{2} \mathrm{H}_{3}$-HEP was formed (Fig. 8).

To achieve a high hydrogenation temperature, but to avoid degeneration of the catalyst ${ }^{18}$, a stock solution containing catalyst and substrate at high concentrations was prepared and kept at room temperature (concentrations: $c_{\text {rhodium }}=21 \mathrm{mM}$, $\left.c_{\text {ligand }}=23 \mathrm{mM}, c_{\mathrm{HEA}}=55.4 \mathrm{mM}\right)$. The reaction chamber was heated in a water bath to $\sim 90-95^{\circ} \mathrm{C}$. Shortly before the experiment, one part $(100 \mu \mathrm{l})$ of the stock and nine parts of hot $\mathrm{H}_{2} \mathrm{O}$ or $\mathrm{D}_{2} \mathrm{O}\left(\sim 90^{\circ} \mathrm{C}\right)$ were filled into the reaction chamber and placed in the MRI scanner. Unless otherwise indicated, the final concentration was $2.1 \mathrm{mM}$ for the catalyst (with $10 \%$ excess of the ligand) and $5.54 \mathrm{mM}$ of HEA.

For the experiment with elevated tracer concentrations, the required amount of HEA was added to the reactor afterwards (for example $9 \mathrm{mg}$ to reach $80 \mathrm{mM}$ in $1 \mathrm{ml}$ solvent).

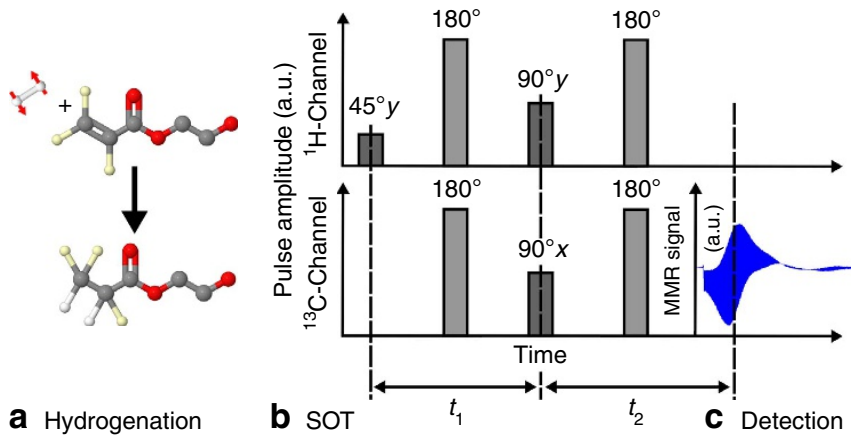

Figure 8 | Schematic view of the HP method: The tracer to-behyperpolarized is formed by catalytic addition of $\mathrm{pH}_{2}$ to an unsaturated precursor molecule (a, here: hydroxyethyl-acrylate forms the angiographytracer $\left.^{27,30} \mathrm{HEP}\right)$. Next, a pulse sequence is applied subsequently to transfer the spin order (grey bars, here: PH-INEPT + (ref. 45)), and the enhanced ${ }^{13} \mathrm{C}$ signal is recorded (c). In $\mathbf{b}$, refocusing pulses are shown in light grey. (c) Real ${ }^{13} \mathrm{C}$-data in the time domain acquired after one HP experiment.

This procedure permitted obtaining a high hydrogenation temperature of $>80^{\circ} \mathrm{C}$, while keeping the catalyst at room temperature for most of the time ${ }^{18}$.

Spin order transfer. Both PH-INEPT + (ref. 45) and l-PH-INEPT + (ref. 31) sequences were implemented on the MRI system. The PH-INEPT + sequence, which consists of three excitation pulses $\left(45^{\circ} \mathrm{y}^{1}{ }^{1} \mathrm{H}, 90^{\circ} \mathrm{y}^{-1} \mathrm{H}\right.$ and $\left.90^{\circ} \mathrm{x}^{-1}{ }^{13} \mathrm{C}\right)$ interleaved by two refocusing pulses $\left(180^{\circ}\right.$ on ${ }^{1} \mathrm{H}$ and $\left.{ }^{13} \mathrm{C}\right)$, was used to transform the para-spin order into hyperpolarized transversal ${ }^{13} \mathrm{C}$ magnetization (Fig. 8). The transversal magnetization generated by this method is short-lived and decays with $T_{2}{ }^{*}$. The l-PH-INEPT + (ref. 31) sequence, instead, generates long-lasting longitudinal magnetization by the addition of an extra $90^{\circ} \mathrm{y}^{13} \mathrm{C}$ pulse to $\mathrm{PH}$ INEPT + . Note that the evolution intervals between the pulses of the sequences $\left(t_{1}\right.$ and $t_{2}$ ) depend on the hydrogen-hydrogen- and hydrogen-carbon $J$-couplings of the target molecule.

Quantum mechanical simulations were carried out to optimize $t_{1}$ and $t_{2}$ and to determine the theoretical polarization yield. The simulations were performed using the product operator and density matrix formalism in a reduced spin system containing the two para-hydrogens and the target nucleus ${ }^{13} \mathrm{C}(J$-couplings: $\left.J_{\mathrm{H}-\mathrm{H}}=7.57 \mathrm{~Hz}, J_{\mathrm{H} 1-\mathrm{C}}=7.24 \mathrm{~Hz} J_{\mathrm{H} 2-\mathrm{C}}=-5.62 \mathrm{~Hz}\right)^{46}$. It was assumed that only longitudinal spin order survives the hydrogenation process, and that evolution takes place under the isotropic liquid-state Hamiltonian. The optimal timings with respect to the highest polarization yield were obtained by numerical optimization. Relaxation was neglected and rotation operators were applied to mimic the effect of radio frequency pulses. To assess the effect of erroneous intervals, flip angles and $J$-couplings, these parameters were varied ${ }^{31,47}$.

MR settings. For in vitro experiments, $1 \mathrm{ml}$ of $\mathrm{H}_{2} \mathrm{O}$ was filled into the reactor and placed in the isocentre of the scanner (referred to as position 1) to match and tune the coil and to adjust the frequency and field homogeneity (iterative first-order shim). The ${ }^{1} \mathrm{H}$ frequency was manually set to $\sim 2.8$ p.p.m. The ${ }^{13} \mathrm{C}$ frequency was set to $\sim 185$ p.p.m., the chemical shift of $1-{ }^{13} \mathrm{C} \mathrm{HEP}$, using the ${ }^{13} \mathrm{CO}$-resonance of acetone (referred to as model solution $\mathrm{M} 1,1.1$ atom \% natural abundance of carbon-13,8 ml, concentration of $\sim 14 \mathrm{M}$, chemical shift of the ${ }^{13} \mathrm{CO}$ resonance 210 p.p.m.).

The ${ }^{13} \mathrm{C}$ flip angle was calibrated manually using free induction decays with varying pulse power and an $8 \mathrm{ml}$ sample of ethanol at natural abundance (reference pulse gains for a $1 \mathrm{~ms}, 90^{\circ}$ pulse of $53.6 \mathrm{~W}(17.2 \mathrm{~dB})$ for ${ }^{1} \mathrm{H}$ and $163.5 \mathrm{~W}(8.2 \mathrm{~dB})$ for $\left.{ }^{13} \mathrm{C}\right)$.

For the imaging experiments, the reactor was placed at a distance of $3.5 \mathrm{~cm}$ to the isocentre (referred to as position 2). The ${ }^{1} \mathrm{H}$ and ${ }^{13} \mathrm{C}$ flip angles were calibrated using a model solution containing $667 \mathrm{mg} 1-{ }^{13} \mathrm{C}$ sodium acetate in $2 \mathrm{ml}$ deionized $\mathrm{H}_{2} \mathrm{O}$ (denoted as model solution $\mathrm{M} 2$, longitudinal relaxation time $T_{1}=(22 \pm 1) \mathrm{s}$ (see Supplementary Fig. 6), 99 atom $\%{ }^{13} \mathrm{C}, \mathrm{MW}=83.03 \mathrm{~g} \mathrm{~mol}^{-1}, c=4 \mathrm{M}$, CAS 23424-28-4, Sigma Aldrich, USA) in a cutoff $10 \mathrm{~mm}$ nuclear magnetic resonance (NMR) tube that was placed within the reaction chamber. Compared with the flip angles measured at position 1 , deviations of $1 \%$ for ${ }^{1} \mathrm{H}$ and $18 \%$ for ${ }^{13} \mathrm{C}$ were found (reference pulse gains of $54.2 \mathrm{~W}(17.1 \mathrm{~dB})$ for ${ }^{1} \mathrm{H}$ and $190.5 \mathrm{~W}(7.1 \mathrm{~dB})$ for ${ }^{13} \mathrm{C}$ (Supplementary Fig. 7).

Before HP, the first-order shims and frequencies were adjusted for the reactor and the rat using a PRESS ${ }^{48}$ localization. Both settings were saved and loaded before HP or imaging was conducted.

The receiver gain was set to $64 \mathrm{~dB}$ for all ${ }^{13} \mathrm{C}$-experiments. 
Hyperpolarization. After the reactor was filled with the precursor solution, closed and placed in the magnet, the automated HP procedure was started, consisting of the following steps: First, the hydrogenation reaction was initialized by injecting $p \mathrm{H}_{2}$ at $15 \mathrm{bar}$ (unless indicated otherwise) for a period of $2 \mathrm{~s}$, followed by a variable delay of 1-12 s, yielding a total hydrogenation time of $t_{\mathrm{h}}=3-14 \mathrm{~s}$. Next, the SOT-sequence was executed $(\sim 100 \mathrm{~ms})$. If the PH-INEPT + sequence was used, the ${ }^{13} \mathrm{C}$ signal was detected directly at the end of $t_{2}$; if l-PH-INEPT + was applied, the magnetization was read out after another delay $t_{\mathrm{d}}$, either by non-localized spectroscopy or imaging. Note that $\sim 300 \mu \mathrm{l}$ of the solution was flushed into the outlet tube during the hydrogenation.

Longitudinal relaxation time $\boldsymbol{T}_{\mathbf{1}}$. After an l-PH-INEPT + experiment, the $T_{1}$ of HEP in $\mathrm{D}_{2} \mathrm{O}$ and $\mathrm{H}_{2} \mathrm{O}$ was measured by probing the decaying longitudinal magnetization with small-angle acquisitions of $9^{\circ}$, interleaved by $15 \mathrm{~s}\left(\mathrm{D}_{2} \mathrm{O}\right)$ or $5 \mathrm{~s}\left(\mathrm{H}_{2} \mathrm{O}\right) . T_{1}$ was extracted from an exponential decay function $S(t)=S_{0} \cdot \exp \left(-t / T_{1}\right) \cdot \cos (\alpha)^{n-1}$, where $S(t)$ is the acquired signal, $S_{0}$ is the signal at $t=0, \alpha$ the flip angle $\left(9^{\circ}\right)$ and $n$ the number of the pulse of the series. The absolute polarization of this scan was estimated using the signal of the first acquisition and $\alpha$. Note, that the data points in Fig. 5 were corrected for polarization loss induced by the previous excitations by dividing the measured signals by $\cos (\alpha)^{n-1}$.

Quantification of HP. The absolute polarization yield $P$ was quantified with respect to the signal of a thermally polarized sample (M1) for the in vitro experiments, and a model solution containing $333 \mathrm{mg} 1{ }^{13} \mathrm{C}$ sodium acetate dissolved in $1.2 \mathrm{ml}$ deionized $\mathrm{H}_{2} \mathrm{O}$ (denoted as model solution $\mathrm{M} 3$, concentration of sodium acetate of $c=3.3 \mathrm{M}$ ) for the ex vivo experiments:

$$
P=P_{\text {therm }} \cdot \frac{N_{\text {ref }}}{N_{\mathrm{HP}}} \frac{F_{13 \mathrm{Cref}}}{F_{13 \mathrm{CHP}}} \frac{c_{\mathrm{ref}}}{c_{\mathrm{HP}}} \frac{V_{\text {ref }}}{V_{\mathrm{HP}}} \frac{S_{\mathrm{HP}}}{S_{\text {ref }}}
$$

where HP and ref indicate a value of the hyperpolarized sample and the reference model solution, $N$ is the number of summated scans ( 1 for all HP experiments), $F_{13 \mathrm{C}}$ is the atomic fraction of the ${ }^{13} \mathrm{C}$ isotope, $c$ is the concentration of the molecule, $V$ is the volume of the sample, $S$ the measured ${ }^{13} \mathrm{C}$-NMR signal and $\alpha$ the excitation flip angle, $P_{\text {therm }}$ is the thermal ${ }^{13} \mathrm{C}$-polarization at $7 \mathrm{~T}$ and room temperature ( $\sim 6$ p.p.m.). Note that $100 \% \mathrm{pH}_{2}$ enrichment and complete hydrogenation was assumed, which results in an underestimation of the HP that was actually achieved.

The NMR signals were numerically integrated after multiplication with an exponential function $(10 \mathrm{~Hz})$, Fourier-transformation and automated phase and baseline correction (Topspin 2.0, Bruker, Germany).

Hydrogenation. The quantification of the hydrogenation yield by NMR of the samples after HP is problematic because the hydrogenation reaction continues after HP. Indeed, complete conversion was found in all samples investigated. Attempts to monitor the conversion during the reaction failed because of low signal (localized spectroscopy, PRESS at $9.4 \mathrm{~T}$ and $\sim 100 \mathrm{mM}$ substrate concentration) or strong distortions of the resonances (non-localized spectroscopy).

Instead, the HP yield $(P)$ was recorded as a function of the hydrogenation time $t_{\mathrm{h}}$ at otherwise identical conditions. From the resulting data, an apparent paraorder relaxation time $T_{\text {para }}$ and a hydrogenation constant $T_{\text {cat }}$ were obtained: we hypothesize that $P$ is governed by a saturated growth described by $T_{\text {cat }}$ and a monoexponential decay of the para-order $\left(T_{\text {para }}\right)$ after the hydrogenation occurred. By solving the differential equations describing the chemical kinetics, an analytical expression for $P\left(t_{\mathrm{h}}\right)$ was derived and fitted to the experimental data. The function $P\left(t_{\mathrm{h}}\right)$ reads:

$$
P\left(t_{\mathrm{h}}\right)=\frac{b}{T_{\text {cat }} / T_{\text {para }}-1}\left[\exp \left(\frac{-\left(t_{\mathrm{h}}-t_{0}\right)}{T_{\text {cat }}}\right)-\exp \left(\frac{-\left(t_{\mathrm{h}}-t_{0}\right)}{T_{\text {para }}}\right)\right] .
$$

The coefficients $b$ and $t_{0}$ allow the maximum polarization level to be adjusted and a time delay between the application of $p \mathrm{H}_{2}$ pressure and the onset of the hydrogenation reaction, respectively.

Ex vivo experiments. An ex vivo rat (rattus rattus, $35 \mathrm{~g}, 7 \mathrm{~cm}, 7$ days old) and model solution M3 were placed next to the reaction chamber. An injection hose ( $42 \mathrm{~cm}, 1 / 16$ ", PTFE, SCP GmbH, Germany) was connected to the outlet of the reactor and inserted into the thorax of the rat. The setup was positioned in the coil within the MRI system. The reactor was at a distance of $3.5 \mathrm{~cm}$ from the isocentre (position 2). The field homogeneity was optimized for two volumes, the position of the reactor and the entire rat. The settings were saved and loaded when needed. After the HP, $p \mathrm{H}_{2}$ pressure was released, and a manual valve (V in Fig. 7 ) was actuated to inject the HP-tracer directly into the rat without leaving the magnet.

Two-dimensional single-shot RARE (rapid acquisition with relaxation enhancement $)^{49}$ images were acquired before, as well as $\sim 10 \mathrm{~s}$ and $\sim 41 \mathrm{~s}$ after the HP was generated $\left(90 / 180^{\circ}\right.$, RARE factor 38, partial Fourier factor $1.7778,38.96$ matrix, FOV $(8.4 \mathrm{~cm})^{2}$, zero-filled to $128 \cdot 128,(0.65 \mathrm{~mm})^{2}$ in-plane resolution, 6 -cm-slice thickness, acquisition time $/ T_{\mathrm{R}}=0.487 \mathrm{~s}, T_{\mathrm{E}}=79 \mathrm{~ms}$, acquisition time $487 \mathrm{~ms}$, bandwidth $10 \mathrm{kHz}$, centred in the isocentre).

Subsequently, a $T_{2}$-weighted, two-dimensional, ${ }^{1} \mathrm{H}$-Turbo-RARE sequence was acquired with the same in-plane FOV for anatomical reference $\left(90 / 180^{\circ}\right.$, RARE factor 8, bandwidth $46.9 \mathrm{kHz}$, matrix $256^{2}$, in-plane resolution $(328 \mu \mathrm{m})^{2}$, $T_{\mathrm{R}}=2.5 \mathrm{~s}, T_{\mathrm{E}}=33 \mathrm{~ms}$, acquisition time $80 \mathrm{~s}, 15$ slices $1 \mathrm{~mm}$ thick, $1.5 \mathrm{~mm}$ apart, centred in the isocenter).

A ZTE MRI was acquired to display the reaction chamber and rat simultaneously $\left(2.3^{\circ}\right.$, matrix $128^{3}$, FOV $16.8 \mathrm{~cm}, T_{\mathrm{R}}=4 \mathrm{~ms}$, acquisition time $208 \mathrm{~s}$, bandwidth $300 \mathrm{kHz}, 51,896$ projections, centred in the isocentre).

The SNR was calculated by dividing the maximum signal intensity of a chosen ROI by the standard deviation of the noise (paravision 5.1, Bruker, Germany) (Supplementary Fig. 2 and Supplementary Table 1). For the co-registration, black was set transparent in the ${ }^{13} \mathrm{C}$ image (Fig. 7) (GNU image manipulation program). The ZTE data are shown as a max. intensity projection (image ${ }^{50}$ ). The images were composed with Inkscape.

Data availability. The authors declare that all relevant data presented here are available from the corresponding author upon reasonable request.

\section{References}

1. Kurhanewicz, J. et al. Analysis of cancer metabolism by imaging hyperpolarized nuclei: prospects for translation to clinical research. Neoplasia 13, 81-97 (2011).

2. Xu, V. et al. MR spectroscopy in diagnosis and neurological decision-making. Semin. Neurol. 28, 407-422 (2008).

3. Ardenkjaer-Larsen, J. H. et al. Dynamic nuclear polarization polarizer for sterile use intent. NMR Biomed. 24, 927-932 (2011).

4. Nelson, S. J. et al. Metabolic imaging of patients with prostate cancer using hyperpolarized [1-13C]pyruvate. Sci. Transl. Med. 5, 108 (2013).

5. Cunningham, C. H. et al. Hyperpolarized 13C metabolic MRI of the human heart-initial experience. Circ. Res. 119, 1177-1182 (2016).

6. Ardenkjær-Larsen, J. H. et al. Increase in signal-to-noise ratio of $>10,000$ times in liquid-state NMR. Proc. Natl Acad. Sci. USA 100, 10158-10163 (2003).

7. Bowers, C. R. \& Weitekamp, D. P. Parahydrogen and synthesis allow dramatically enhanced nuclear alignment. J. Am. Chem. Soc. 109, 5541-5542 (1987).

8. Eisenschmid, T. C. et al. Para hydrogen induced polarization in hydrogenation reactions. J. Am. Chem. Soc. 109, 8089-8091 (1987).

9. Adams, R. W. et al. Reversible interactions with para-hydrogen enhance NMR sensitivity by polarization transfer. Science 323, 1708-1711 (2009).

10. Comment, A. et al. Producing over $100 \mathrm{ml}$ of highly concentrated hyperpolarized solution by means of dissolution DNP. J. Magn. Reson. 194, 152-155 (2008).

11. Batel, M. et al. A multi-sample $94 \mathrm{GHz}$ dissolution dynamic-nuclearpolarization system. J. Magn. Reson. 214, 166-174 (2012).

12. Bornet, A. et al. Boosting dissolution dynamic nuclear polarization by cross polarization. J. Phys. Chem. Lett. 4, 111-114 (2013).

13. Shchepin, R. V., Coffey, A. M., Waddell, K. W. \& Chekmenev, E. Y. PASADENA Hyperpolarized 13C Phospholactate. J. Am. Chem. Soc. 134, 3957-3960 (2012).

14. Shchepin, R. V., Pham, W. \& Chekmenev, E. Y. Dephosphorylation and biodistribution of 1-13C-phospholactate in vivo. J. Label. Compd. Radiopharm. 57, 517-524 (2014).

15. Shchepin, R. V., Coffey, A. M., Waddell, K. W. \& Chekmenev, E. Y. Parahydrogen induced polarization of 1-13C-phospholactate-d2 for biomedical imaging with $>30,000,000$-fold NMR Signal Enhancement in Water. Anal. Chem. 86, 5601-5605 (2014).

16. Reineri, F., Boi, T. \& Aime, S. ParaHydrogen Induced Polarization of 13C carboxylate resonance in acetate and pyruvate. Nat. Commun. 6, 5858 (2015).

17. Hövener, J.-B. et al. PASADENA hyperpolarization of $13 \mathrm{C}$ biomolecules: equipment design and installation. Magn. Reson. Mater. Phys. 22, 111-121 (2009).

18. Kadlecek, S. et al. A simple and low-cost device for generating hyperpolarized contrast agents using parahydrogen. NMR Biomed. 24, 33-42 (2011).

19. Waddell, K. W., Coffey, A. M. \& Chekmenev, E. Y. In situ detection of PHIP at $48 \mathrm{mT}$ : demonstration using a centrally controlled polarizer. J. Am. Chem. Soc. 133, 97-101 (2011).

20. Wagner, S., Agraz, J. \& Li, D. Devices and methods for parahydrogen induced polarization. US patent 2,217,262 (2015).

21. Agraz, J., Grunfeld, A. M., Cunningham, K., Li, D. \& Wagner, S. Improved PHIP polarization using a precision, low noise, voltage controlled current source. J. Magn. Reson. 77-84 (2013).

22. Agraz, J. et al. LabVIEW-based control software for para-hydrogen induced polarization instrumentation. Rev. Sci. Instrum. 85, 044705 (2014).

23. Agraz, J. et al. PHIP instrumentation pinch valve system for sample delivery, process and collection. Adv. Biomed. Sci. Eng. 1, 8-18 (2014).

24. Comment, A. et al. Principles of operation of a DNP prepolarizer coupled to a rodent MRI scanner. Appl. Magn. Reson. 34, 313-319 (2008).

25. Jannin, S. et al. A $140 \mathrm{GHz}$ prepolarizer for dissolution dynamic nuclear polarization. J. Chem. Phys. 128, 241102 (2008) 
26. Hövener, J.-B. et al. Quality assurance of PASADENA hyperpolarization for 13C biomolecules. Magn. Reson. Mater. Phys. 22, 123-134 (2009).

27. Goldman, M., Johannesson, H., Axelsson, O. \& Karlsson, M. Design and implementation of $\mathrm{C}-13$ hyperpolarization from para-hydrogen, for new MRI contrast agents. Comptes Rendus Chim. 9, 357-363 (2006).

28. Johannesson, H., Axelsson, O. \& Karlsson, M. Transfer of para-hydrogen spin order into polarization by diabatic field cycling. C R Phys. 5, 315-324 (2004).

29. Coffey, A. M. et al. Open-source automated parahydrogen hyperpolarizer for molecular imaging using 13C metabolic contrast agents. Anal. Chem. 88, 8279-8288 (2016)

30. Olsson, L. E. et al. MR coronary angiography in pigs with intraarterial injections of a hyperpolarized 13C substance. Magn. Reson. Med. 55, 731-737 (2006).

31. Dutta, P., Martinez, G. V. \& Gillies, R. J. A new horizon of DNP technology: application to in-vivo $13 \mathrm{C}$ magnetic resonance spectroscopy and imaging. Biophys. Rev 5, 271-281 (2013).

32. Bär, S. et al. On the spin order transfer from parahydrogen to another nucleus. J. Magn. Reson. 225, 25-35 (2012).

33. Weiger, M., Pruessmann, K. P. \& Hennel, F. MRI with zero echo time: hard versus sweep pulse excitation. Magn. Reson. Med. 66, 379-389 (2011).

34. Koptyug, I. V. et al. Para-hydrogen-induced polarization in heterogeneous hydrogenation reactions. J. Am. Chem. Soc. 129, 5580-5586 (2007).

35. Kovtunov, K. V., Beck, I. E., Bukhtiyarov, V. I. \& Koptyug, I. V. Observation of parahydrogen-induced polarization in heterogeneous hydrogenation on supported metal catalysts. Angew Chem. Int. Ed. Engl. 47, 1492-1495 (2008).

36. Kovtunov, K. V., Zhivonitko, V. V., Corma, A. \& Koptyug, I. V. Parahydrogen-induced polarization in heterogeneous hydrogenations catalyzed by an immobilized $\mathrm{Au}(\mathrm{III})$ complex. J. Phys. Chem. Lett. 1, 1705-1708 (2010).

37. Glöggler, S. et al. A nanoparticle catalyst for heterogeneous phase parahydrogen-induced polarization in water. Angew. Chem. Int. Ed. Engl. 54, 2452-2456 (2015)

38. Reineri, F. et al. Use of labile precursors for the generation of hyperpolarized molecules from hydrogenation with parahydrogen and aqueous-phase extraction. Angew Chem. Int. Ed. Engl. 50, 7350-7353 (2011).

39. Comment, A. \& Van Der Klink, J. Infusion pump with phase separator. US patent 8,034,027 (2011).

40. Chekmenev, E. Y. et al. PASADENA hyperpolarization of succinic acid for MRI and NMR spectroscopy. J. Am. Chem. Soc. 130, 4212-4213 (2008).

41. Bhattacharya, P. et al. Towards hyperpolarized 13C-succinate imaging of brain cancer. J. Magn. Reson. 186, 150-155 (2007).

42. Ross, B. D., Bhattacharya, P., Wagner, S., Tran, T. \& Sailasuta, N. Hyperpolarized MR imaging: neurologic applications of hyperpolarized metabolism. Am. J. Neuroradiol. 31, 24-33 (2010).

43. Zacharias, N. M., Chan, H. R., Sailasuta, N., Ross, B. D. \& Bhattacharya, P. Real-time molecular imaging of tricarboxylic acid cycle metabolism in vivo by hyperpolarized 1-13C diethyl succinate. J. Am. Chem. Soc. 134, 934-943 (2012).

44. Hoevener, J.-B. et al. A continuous-flow, high-throughput, high-pressure parahydrogen converter for hyperpolarization in a clinical setting. NMR Biomed. 26, 124-131 (2013).

45. Haake, M., Natterer, J. \& Bargon, J. Efficient NMR pulse sequences to transfer the parahydrogen-induced polarization to hetero nuclei. J. Am. Chem. Soc. 118, 88-91 (1996).
46. Goldman, M. \& Johannesson, H. Conversion of a proton pair para order into 13C polarization by rf irradiation, for use in MRI. Comptes Rendus Phys. 6, 575-581 (2005).

47. Hövener, J.-B., Knecht, S., Schwaderlapp, N., Hennig, J. \& von Elverfeldt, D. Continuous re-hyperpolarization of nuclear spins using parahydrogen: theory and experiment. Chem. Phys. Chem. 15, 2451-2457 (2014).

48. Bottomley, P. A. Spatial localization in NMR spectroscopy in vivo. Ann. N. Y. Acad. Sci. 508, 333-348 (1987).

49. Hennig, J., Nauerth, A. \& Friedburg, H. RARE imaging: a fast imaging method for clinical MR. Magn. Reson. Med. 3, 823-833 (1986).

50. Schneider, C. A., Rasband, W. S. \& Eliceiri, K. W. NIH Image to ImageJ: 25 years of image analysis. Nat. Methods 9, 671-675 (2012).

\section{Acknowledgements}

Funding support by the DFG (HO 4604/1-1 and HO 4604/2-1), the German Consortium for Translational Cancer Research, the European Union's Horizon 2020 research and innovation programme under the Marie Sklodowska-Curie grant agreement No 642773 and the contribution of the COST Action TD1103 is gratefully acknowledged.

\section{Author contributions}

A.B.S. and S.B. have contributed equally and have performed the experiments. A.B.S., S.B. and T.L. have implemented the method. J.-B.H., T.L., N.S., C.M., A.D., P.R., S.K. and J.G.S. helped with measurements. A.B.S., T.L. and W.S. designed the reactor. W.S. constructed the reactor. The experiment was devised by J.-B.H. J.H., D.v.E. and J.-B.H. supervised the project and provided the equipment. A.B.S., J.-B.H. and S.B. evaluated and illustrated the results. All authors contributed to the discussion and interpretation of the results. A.B.S. and J.-B.H. wrote the manuscript.

\section{Additional information}

Supplementary Information accompanies this paper at http://www.nature.com/ naturecommunications

Competing financial interests: The authors declare no competing financial interests.

Reprints and permission information is available online at http://npg.nature.com/ reprintsandpermissions/

How to cite this article: Schmidt, A. B. et al. Liquid-state carbon-13 hyperpolarization generated in an MRI system for fast imaging. Nat. Commun. 8, 14535 doi: $10.1038 /$ ncomms14535 (2017)

Publisher's note: Springer Nature remains neutral with regard to jurisdictional claims in published maps and institutional affiliations.

This work is licensed under a Creative Commons Attribution 4.0 International License. The images or other third party material in this article are included in the article's Creative Commons license, unless indicated otherwise in the credit line; if the material is not included under the Creative Commons license, users will need to obtain permission from the license holder to reproduce the material To view a copy of this license, visit http://creativecommons.org/licenses/by/4.0/

(C) The Author(s) 2017 